La revue La revue pour l'histoire du CNRS

POUR LHISTORE Du CNRS $\quad 5 \mid 2001$

Des laboratoires à l'étranger

\title{
La mission du CNRS en Allemagne (1945-1950)
}

Entre exploitation et contrôle du potentiel scientifique allemand

Corine Defrance

\section{(2) OpenEdition}

Journals

Édition électronique

URL : https://journals.openedition.org/histoire-cnrs/3372

DOI : $10.4000 /$ histoire-cnrs.3372

ISSN : 1955-2408

Éditeur

CNRS Éditions

Édition imprimée

Date de publication : 5 novembre 2001

ISBN : 978-2-271-05925-3

ISSN : $1298-9800$

Référence électronique

Corine Defrance, «La mission du CNRS en Allemagne (1945-1950)», La revue pour I'histoire du CNRS

[En ligne], 5 | 2001, mis en ligne le 20 juin 2007, consulté le 20 mai 2021. URL : http://

journals.openedition.org/histoire-cnrs/3372 ; DOI : https://doi.org/10.4000/histoire-cnrs.3372

Ce document a été généré automatiquement le 20 mai 2021

Comité pour l'histoire du CNRS 


\title{
La mission du CNRS en Allemagne (1945-1950)
}

\author{
Entre exploitation et contrôle du potentiel scientifique allemand
}

\section{Corine Defrance}

1 Un an et demi après avoir mis en place une mission en Allemagne, le Centre national de la recherche scientifique, alors dirigé par le généticien Georges Teissier, rédigea un important rapport sur cette mission daté du 25 novembre 1946. Après avoir rappelé sa mise en place et ses objectifs, ce compte rendu dressait un bilan provisoire des résultats obtenus, notamment en ce qui concernait la récupération et l'achat de matériel scientifique. Document interne au CNRS, ce " compte rendu sur la mission du CNRS en Allemagne", signé par Marcel Autheman, chef du service des affaires allemandes du CNRS, repose en grande partie sur les rapports de Louis Cagniard, chef de la mission en Allemagne depuis le début de l'année 1946. Au moment de la rédaction de ce rapport, L. Cagniard avait déjà quitté ses fonctions (en juillet) et avait été remplacé à la tête de la mission par Jean Decombe, professeur de chimie à l'université de Dijon.

2 L'instauration de cette mission française, qui devait perdurer jusqu'en 1950, constitue l'un des aspects de l'immense entreprise de contrôle et d'exploitation de la science et des savants allemands dans laquelle s'étaient lancées les quatre puissances d'occupation en Allemagne - Union soviétique, États-Unis, Grande-Bretagne et France -, encore alliées en 1945, mais déjà rivales avant même que la guerre froide naissante ne vînt les diviser. Opposant toujours plus radicalement l'URSS aux Britanniques et Américains, puis bientôt aux Français dont la position d'arbitre entre alliés oriental et occidentaux s'avéra vite intenable, la confrontation idéologique Est/Ouest ne supprima pas pour autant toute forme de concurrence entre les trois Occidentaux dans la course au matériel scientifique et aux savants allemands.

3 Aussi, à travers la mise en place, le fonctionnement et le bilan de la mission du CNRS en Allemagne, de 1945 à 1950, essaierons-nous d'apprécier les enjeux que la science allemande représentait pour la recherche française et la singularité de la politique scientifique française en Allemagne.

Les Alliés et la science allemande en 1945 
$4 \mathrm{Au}$ moment où leurs troupes pénétrèrent sur le sol allemand, les Alliés ignoraient presque complètement l'état d'avancement de la science allemande. Tous savaient qu'avant guerre, la recherche allemande avait été en pointe dans de nombreux secteurs comme la chimie ou la science de l'atome. Les savants allemands émigrés, outre la perte de potentiel scientifique que leur exil avait induite pour le régime nazi, avaient certes informé Britanniques et Américains des travaux en cours dans le Reich, mais, avec la guerre, le contact avec la science allemande fut rompu: les chercheurs allemands cessèrent de participer aux congrès internationaux, leurs travaux ne furent plus publiés et ceux qui s'étaient mis au service du régime travaillaient dans le secret. Les Alliés savaient que l'économie de guerre avait stimulé la recherche et les innovations allemandes dans bien des domaines: production de caoutchouc artificiel et d'essence synthétique, ce qui avait permis à Hitler de se lancer dans une guerre de conquête sans crainte d'être privé des matières premières dont le Reich ne disposait pas; progrès spectaculaires en matière d'armement liés notamment aux progrès de la balistique (les V1 et V2) ; préparation d'une arme secrète dont Hitler annonçait l'emploi imminent. Il faut garder à l'esprit que, lors de l'entrée des forces alliées sur le territoire du Reich, la guerre n'était terminée ni contre l'Allemagne ni contre le Japon, et que les Américains n'avaient pas encore achevé de mettre au point leur bombe atomique ${ }^{1}$. Dans ce contexte, la première des priorités fut de s'informer de la position exacte de l'Allemagne dans la course aux armements. Pour les Américains, il s'agissait avant tout de savoir si les Allemands préparaient une bombe atomique et quel était le degré d'avancement de leur programme. Ce fut la tâche de la mission $\mathrm{ALSOS}^{2}$, contrôlée par les militaires (le général Leslie Groves) et composée de savants spécialistes de l'atome ${ }^{3}$.

Chacun des Alliés voulait savoir où en était la recherche allemande, dans tous les secteurs, et mit sur pied des unités spéciales, qui accompagnaient les troupes les plus avancées sur tous les fronts. Les Français ne dérogèrent pas à la règle. Une section $\mathrm{T}$ fut rattachée au deuxième bureau d'état-major de la Première Armée conduite par le général de Lattre de Tassigny. Dirigée par le colonel Gaston de Verbigier de Saint-Paul, elle regroupait des spécialistes militaires chargés de découvrir et d'exploiter toutes les usines et centres de recherche pouvant intéresser la Défense nationale ${ }^{4}$. En juillet 1945, lors de la dissolution de la Première Armée, la section $\mathrm{T}$ fut remplacée par une section française d'information scientifique, établie sur le modèle des Field Information Agencies for Technics (FIAT) britannique et américaine. La section française - généralement appelée French FIAT - resta dirigée par G. de Verbigier de Saint-Paul.

6 Ces unités scientifiques alliées visaient globalement les mêmes objectifs: outre l'information sur l'avancement des travaux, il s'agissait de veiller au désarmement allemand et d'empêcher tout réarmement, de tirer profit de la recherche allemande (matériel, savants et techniciens, brevets) et d'éviter, pour les Britanniques et les Américains, que les Soviétiques ne s'emparent du potentiel scientifique allemand (humain et technique). Ainsi, au cours des semaines qui précédèrent la capitulation, les Américains avaient-ils préventivement bombardé des centres militaires de recherche et de production avant que l'Armée rouge ne les atteignît. En juillet, lors du partage définitif de l'Allemagne en zones d'occupation, les Américains ramenèrent de Thuringe et de Saxe-Anhalt des wagons entiers de matériel et quelque 1500 chercheurs et ingénieurs, avant de remettre ces régions à l'administration militaire soviétique en Allemagne $^{5}$. La mission ALSOS eut recours à des méthodes similaires pour empêcher les Français de tirer parti des installations de Haigerloch (embryon de pile atomique ${ }^{6}$ ) et 
des plus grands savants de l'atome qui s'étaient établis à Hechingen dans le Wurtemberg-Hohenzollern. Ayant appris que la région de Hechingen allait faire partie de la zone française d'occupation, ALSOS monta une opération (baptisée Humbug) pour s'emparer de Otto Hahn et de Werner Heisenberg ${ }^{7}$. Ces deux savants les intéressaient au plus haut point, le premier pour avoir découvert la fission du noyau atomique en 1938, le second pour ses recherches nucléaires sous le $\mathrm{III}^{\mathrm{e}} \mathrm{Reich}^{8}$. Selon l'historien israélien Michel Bar-Zohar, le général Groves, qui supervisait le travail d'ALSOS depuis les ÉtatsUnis, aurait redouté que Frédéric Joliot, dont les sympathies communistes étaient connues, ne livrât aux Soviétiques les informations qu'il aurait pu obtenir de ces deux savants9 9 Quant à la cave hébergeant le matériel nécessaire à la fabrication d'une pile, elle fut détruite par les Américains quelques jours avant l'arrivée des forces d'occupation françaises ${ }^{10}$.

7 La petite zone attribuée à l'occupant français, au sud-ouest de l'Allemagne, abritait un potentiel scientifique important, concentré essentiellement dans le WurtembergHohenzollern et le sud du pays de Bade. Comme dans le reste de l'Allemagne, les principaux foyers de recherche relevaient de trois grands types d'établissements : les instituts universitaires, les instituts de l'ex-Kaiser-Wilhelm-Gesellschaft (KWG) et les laboratoires industriels. Les deux premiers groupes se consacraient essentiellement à la recherche fondamentale tandis que le troisième était orienté vers la recherche appliquée ${ }^{11}$. La société Kaiser-Wilhelm avait été fondée en 1911 pour donner une nouvelle impulsion à la recherche. Largement financée par les industriels et la bourgeoisie, la KWG devait échapper en partie au contrôle de l'État. Au cours du III ${ }^{\mathrm{e}}$ Reich, elle avait perdu une bonne partie de cette indépendance sans toutefois abandonner la recherche fondamentale au profit exclusif de travaux à caractère militaire ${ }^{12}$. Éprouvant une très grande méfiance à l'égard de cette très puissante société, les quatre Alliés décidèrent en 1946 de la dissoudre ${ }^{13}$. Les différents instituts continuaient à exister, mais le désordre administratif était considérable, et leur survie dépendait de l'intérêt que leur portaient les puissances d'occupation.

Dans les seules régions du Bade-sud et du Wurtemberg-Hohenzollern, on dénombrait au printemps 1945 deux universités implantées à Fribourg et Tübingen et treize instituts de la KWG, soit $26 \%$ environ des chercheurs de la Société ${ }^{14}$. Ces instituts, regroupés à l'origine à Berlin-Dahlem, s'étaient mis à l'abri des bombardements alliés en se repliant vers des régions rurales, mais leur transfert massif vers l'ouest de l'Allemagne indiquait clairement qu'ils avaient surtout tenté d'échapper à la progression de l'armée Rouge ${ }^{15}$. Au nombre des plus prestigieux de ces laboratoires, on comptait l'institut de biologie d'Alfred Kühn (recherches génétiques), celui de biochimie d'Adolf Butenandt (travaillant en particulier sur les hormones de métamorphose des insectes), l'institut de physique de W. Heisenberg et celui de chimie d'O. Hahn dont l'une des sections se consacrait à la chimie nucléaire. Un autre institut de la KWG, à Haigerloch, poursuivait des recherches sur les méthodes de mesures appliquées à la physique nucléaire. Génétique et science nucléaire constituaient donc les deux principaux points forts de la recherche fondamentale allemande en zone française d'occupation. La recherche industrielle portait essentiellement sur la chimie, en raison de l'implantation de la principale usine de l'IG Farben à Ludwigshafen. Dans cette seule ville, l'IG Farben entretenait neuf laboratoires et plus de deux cents chercheurs ${ }^{16}$.

Compte rendu sur la mission du CNRS en Allemagne 
25 novembre 1946;

Première partie : historique et activité d'après les rapports de Mr. Cagniard

La mission a été créée dans la seconde quinzaine de mai 1945. Le chef en fut M. Lwoff assisté de M. Berthelot. Son but était :

De récupérer le plus possible de matériel scientifique, de machines-outils, de matières premières pour le rééquipement des laboratoires. Une mission de l'Enseignement technique, dirigée par M. Couffignal fut accolée à la mission CNRS, l'Enseignement technique ayant un besoin urgent de machines-outils.

De se livrer à une investigation aussi poussée que possible dans les laboratoires scientifiques ou industriels et de faire le point des résultats acquis par les Allemands pendant la guerre.

Un appel du CNRS à tous les laboratoires permit de recruter rapidement un grand nombre de volontaires des diverses disciplines scientifiques. Un accord passé permit d'accorder à ces volontaires des grades d'assimilation; ces officiers et sousofficiers furent habillés tant bien que mal; leur rémunération fut assurée sous forme de frais de mission; le CNRS put mettre deux voitures Matford à la disposition de la mission.

II existait à Lindau, rattaché à l'EM de la première armée, une section “ $\mathrm{T}$ ” qui avait la charge de récupérer du matériel et d'enquêter sur les questions scientifiques intéressant l'Armée.

Les buts de la section $\mathrm{T}$ et de la mission CNRS étaient assez voisins ; et M. Lwoff trouva près du Colonel de Verbigier de St-Paul, chef des techniciens à la section $\mathrm{T}$, l'accueil qui permit à la mission de surmonter les difficultés inévitables résultant du fait qu'elle n'était accréditée nulle part.

La Mission du CNRS fut, non pas rattachée officiellement mais accolée à la section $\mathrm{T}$, ce qui lui permit de se maintenir.

Au mois de juillet 1945, la suppression de la première Armée fit prévoir celle de la Section T. Les autorités du Gouvernement militaire, qui commençaient à s'installer, contestèrent les prérogatives, droits de réquisition et de saisie qui appartenaient à la section T. Les missionnaires du CNRS partaient les uns après les autres, enfin la section $\mathrm{T}$ quittait Lindau pour Offenbourg et s'intégra ensuite dans la FIAT.

$C^{\prime}$ est à cette époque que M. Cagniard prit la suite de M. Lwoff.

N'ayant plus de missionnaires à sa disposition, tous, sauf M. Duval, étant rentrés pour leurs cours ou leur laboratoire, M. Cagniard demandait de lui affecter, non plus des missionnaires temporaires, avec lesquels il n'était pas possible d'entreprendre un travail suivi, mais une dizaine de missionnaires permanents.

Dans un pays dépourvu de communications ferroviaires, postales et télégraphiques, un parc auto bien équipé était la condition indispensable de toute activité. Sur l'initiative du général de St-Paul, un parc de voitures fut constitué et un atelier d'entretien fut équipé sous la direction d'un missionnaire permanent du CNRS. Enfin, un stock d'essence fut constitué.

M. Joliot-Curie donna à la mission les buts suivants :

1. Continuer les récupérations et saisies de matériel quand l'occasion s'en présenterait.

2. Acheter du matériel neuf dans les usines d'Allemagne, matériel intéressant $a$ priori, par ses prix peu élevés et aussi, parce que le marché français ne pouvait le livrer.

3. Commencer à prendre en charge et à contrôler les laboratoires et savants d'Allemagne.

4. Continuer l'information scientifique.

5. Accueillir, aider les investigateurs envoyés en mission temporaire par le CNRS ou d'autres organismes officiels ou privés.

6. Collaborer, au sein de la FIAT en particulier, avec les sous-sections spécifiquement militaires, en les aidant de notre compétence scientifique.

7. Assurer en Autriche les mêmes tâches.

$[\ldots]$ 
Conclusion de la première partie

Les extraits précédents des rapports de Mr. Cagniard montrent d'une façon suffisamment nette, la nécessité pour le CNRS d'entretenir une mission en Allemagne et en Autriche aux points de vue investigation scientifique, contrôle de la recherche, face vis à vis des autres nations alliées, enfin achat de matériel.

II serait en particulier déplorable d'abandonner les travaux entrepris :

FIAT Reviews, revues qui permettent de diffuser en France la totalité des recherches allemandes pendant ces 5 années de guerre ;

Contrôle des laboratoires qui nous sont confiés ;

Possibilité d'envoyer de jeunes chercheurs s'initier aux techniques nouvelles ;

Il y a lieu d'ajouter que pour la préparation des clauses du traité de paix, la mission du Centre a souvent été consultée et continue à l'être pour tout ce qui concerne le désarmement scientifique.

Les textes proposés par la mission ont toujours été adoptés par la Commission du ministère de la Défense nationale.

Il reste à examiner la question du rendement financier de la mission et celle des achats de matériel neuf.

Le bilan du $1^{\mathrm{er}}$ octobre 1946 est approximativement le suivant :

Matériel récupéré supérieur à 100 millions

Matériel acheté et livré au 1. Oct. $46: 8$ millions

Frais généraux : 4 millions

Cf. Archives Nationales F800 284/115.

La mise en place de la mission du CNRS

Alors que les Français disposaient déjà d'une structure militaire pour exploiter et contrôler la recherche allemande, quelles furent les raisons qui motivèrent l'implantation d'une mission du CNRS en Allemagne au lendemain de la capitulation du Reich ? Remarquons tout d'abord qu'il ne s'agit pas de la première mission que le CNRS établit à l'étranger. Pendant la guerre, des chercheurs s'étaient préoccupés de relancer la recherche française sans attendre la victoire alliée: le biochimiste Louis Rapkine s'était employé à renouer les liens entre scientifiques français et savants angloaméricains. Bénéficiant de l'appui du général de Gaulle, un groupe de recherche opérationnelle français, bientôt rattaché au CNRS, avait été fondé à Londres le 15 août 1944. Sa mission principale était de réaliser des synthèses des recherches menées par les Alliés pendant la guerre. A titre d'exemple, la mission Rapkine contribua à introduire la fabrication et l'utilisation de la pénicilline en France. L'un des membres du pool pénicilline, André Lwoff, devait devenir le premier chef de la mission du CNRS en Allemagne quelques mois plus tard ${ }^{17}$. Même si la mission en Allemagne devait répondre à des objectifs spécifiques, le point commun de ces missions mises en place en 1944-1945 (Angleterre, États-Unis, Allemagne) était de s'informer sur l'état d'avancement de la recherche dans les pays d'implantation. Il est évident que le CNRS, au sortir de la guerre, avait un énorme travail de remise à flot à effectuer. Dès sa nomination à la tête de cette institution, en septembre 1944, F. Joliot avait souhaité que la France participât à "la moisson scientifique que laiss[ait] présager la future défaite de l'Allemagne " et fit en sorte que le CNRS ouvrît un " front scientifique de guerre ${ }^{18}$ ".

La coopération entre scientifiques du CNRS et militaires pour l'exploitation de la recherche allemande parut débuter dès les tout premiers jours de l'année 1945. À la mijanvier, le comité de la Défense nationale créa un comité de coordination des recherches scientifiques, présidé par le général Dassault, secondé par F. Joliot ${ }^{19}$. On semblait s'orienter vers une collaboration entre civils et militaires telle que la pratiquaient les trois autres alliés, qu'il s'agit des FIAT anglaise et américaine, 
structures militaires au sein desquelles des scientifiques civils travaillaient de concert avec des militaires, ou des équipes de chercheurs, ingénieurs et techniciens que les Soviétiques envoyaient dans les entreprises, universités et laboratoires allemands sous la protection de l'armée ${ }^{20}$. Le directeur du CNRS, pressé d'instaurer une collaboration concrète, proposa en mars d'adjoindre des agents techniques de liaison aux troupes de première ligne. Mais il ne fut pas donné suite à cette proposition et la mission du CNRS ne put s'établir en Allemagne que dans la seconde moitié de mai $1945^{21}$.

11 En fait, les militaires étaient surtout soucieux de récupérer du matériel, tandis que le CNRS voulait préserver les capacités de fonctionnement de certains laboratoires pour s'informer de l'état d'avancement des travaux et tirer profit des dernières découvertes. F. Joliot déplora ouvertement les destructions et le pillage auxquels s'étaient livrés des militaires, rendant les installations inutilisables ${ }^{22}$. C'est ainsi qu'au printemps 1945 , l'appareil à haute tension de l'institut (KWI) de chimie de Tailfingen avait été démontée ${ }^{23}$. Aussi, malgré la volonté affichée par les militaires de coopérer avec les chercheurs civils, l'armée a sans doute d'abord voulu garder le contrôle de la situation, laissant ses services scientifiques opérer seuls ${ }^{24}$. Trop préoccupée par la récupération du matériel, elle semble, dans certains cas, avoir très gravement négligé les savants eux-mêmes, comme en témoigne le double rapt de O. Hahn et W. Heisenberg. Samuel Goudsmit, avec une mordante ironie, devait commenter: "Les troupes coloniales françaises s'intéressaient plus aux cochons et aux poulets qu'aux savants spécialistes de l'atome ${ }^{25}$." L'envoi d'une mission scientifique du CNRS devait mettre de l'ordre dans cette situation. Ayant conscience de la concurrence dans la " course à la matière grise ", les scientifiques français entendaient tirer profit de la recherche allemande sans se laisser dépouiller du " butin " par leurs alliés ${ }^{26}$.

Dès le mois de mars 1945, F. Joliot s'était mis en quête de scientifiques prêts à partir pour l'Allemagne. Un service des Affaires allemandes, dirigé par M. Autheman, avait été mis en place à Paris au siège du CNRS. II fut en premier lieu chargé de l'envoi des missionnaires et lança un appel aux laboratoires pour recruter des volontaires dans toutes les disciplines. F. Joliot dressa des listes où prédominaient d'une part les chimistes (étant donné l'importance de cette industrie en Allemagne), d'autre part les Alsaciens (pour leur maîtrise de l'allemand). A. Lwoff, de l'Institut Pasteur, fut placé à la tête du premier groupe à partir pour l'Allemagne, avec pour adjoint André Berthelot, physicien dans l'équipe de Joliot au Collège de France. La mission fut alors accolée, mais non intégrée, à la section $\mathrm{T}$ de la Première Armée. Aussi s'établit-elle à Lindau, sur les bords du lac de Constance, où le général de Lattre de Tassigny avait établi son quartier général ${ }^{27}$.

Deux mois plus tard, A. Lwoff devait regagner son laboratoire. La phase d'installation prenait fin et le géophysicien L. Cagniard, alors maître de conférences à l'université de Strasbourg, prit la direction de la mission. Lors de la transformation de la section T, en juillet 1945, la mission du CNRS fut intégrée à la French FIAT. La mission du CNRS, désormais installée à Offenburg comme le reste de la FIAT, disposait de divisions pour les différentes régions de la zone française en Allemagne (Bade-Forêt-Noire, BavièreWurtemberg ${ }^{28}$, projet de sections à Mayence et Coblence) ainsi que d'une antenne à Innsbruck pour la zone française d'occupation en Autriche (Tyrol-Vorarlberg) ${ }^{29}$. L. Cagniard demanda alors au CNRS que lui soient envoyés des missionnaires permanents. En 1946, il put compter sur une équipe d'une vingtaine de permanents, auxquels s'ajoutaient les spécialistes envoyés à titre temporaire pour des opérations précises. 
Ainsi, lorsqu'il fut chargé de la récupération du matériel du laboratoire Wankel (recherches sur les moteurs) à Lindau, R. Vichnievsky se fit seconder par deux ingénieurs et un chef d'atelier de la Station nationale de recherches Claude-Bonnier. Ils étaient chargés de régler les questions du démontage et du transport des machines ${ }^{30}$. En 1945, cent cinquante scientifiques furent envoyés en mission en Allemagne par le CNRS. Les membres de la mission, comme l'ensemble du personnel civil du gouvernement militaire, furent assimilés à des grades militaires, reçurent un uniforme et furent astreints à la discipline militaire ${ }^{31}$.

Les deux objectifs initiaux de la mission furent la récupération de matériel scientifique allemand et la collecte d'informations sur la recherche allemande.

Par la récupération, la mission visait à rééquiper les laboratoires français spoliés pendant la guerre. Le service des affaires allemandes du CNRS se chargeait de centraliser les demandes de matériel émanant des centres français. En Allemagne, tout laboratoire ayant travaillé pour la guerre pouvait être considéré comme une prise de guerre. Le matériel récupéré était expédié sur Paris aux laboratoires de Bellevue, le plus souvent par chemin de fer (accord avec la SNCF), inventorié puis réparti par le service central ${ }^{32}$. Parfois, il ne s'agissait pas tant de récupération ou de compensation que d'acquisition à bon compte. Par ailleurs, la mission était aussi chargée d'attribuer du matériel aux établissements de l'enseignement technique. Un des chercheurs de la mission, le mathématicien Louis Couffignal, de l'institut Henri-Poincaré, était préposé à la récupération et à l'attribution de machines-outils pour l'enseignement technique. Les autorisations d'enlèvement de matériel furent accordées très généreusement par les militaires de la section T à la mission du CNRS. En juin 1945, la mission tenta de récupérer au plus vite du matériel dans les laboratoires situés dans les secteurs occupés par la Première Armée qui devaient être restitués aux Alliés. Ainsi R. Vichnievsky fut-il chargé du centre de recherche sur les moteurs de l'usine Hirth de Stuttgart. Dans son rapport, il regretta que cette opération ait été menée si tardivement, au moment où le repli des Français compliquait considérablement l'expédition du matériel expérimental vers la France ${ }^{33}$. Citons aussi le cas du KWI für Metallforschung (Institut de recherches sur les métaux) que la mission du CNRS transféra de Stuttgart à Constance (zone française) où il poursuivit ses travaux sous la direction d'un ingénieur français du ministère de l'Air ${ }^{34}$. L'information sur la recherche allemande revêtait deux aspects : d'une part l'état des laboratoires allemands (leurs possibilités financières et matérielles); d'autre part l'état d'avancement de la recherche en Allemagne ${ }^{35}$. Le numéro deux de la mission du CNRS, le physicien Robert Guillien, avec ses collègues britanniques et américains, aida les chercheurs allemands à publier dans les FIAT Reviews leurs travaux menés au cours de la période 1939-1945. Loin d'être seulement une faveur accordée aux savants allemands, la publication de ces revues permettait de diffuser en France les résultats de la recherche allemande des cinq années de guerre ${ }^{36}$.

Les objectifs à prospecter étaient déterminés par le service des Affaires allemandes de la centrale du CNRS. Ce service devait aussi assurer la diffusion des renseignements recueillis aux instances intéressées (Air, Marine, Guerre, État-major de la Défense nationale $)^{37}$. La mission d'investigation du CNRS en Allemagne ne se limitait pas à la recherche fondamentale. Les chercheurs pouvaient et devaient également enquêter dans les laboratoires industriels. Il semble qu'entre la mission T, puis French FIAT et la mission du CNRS, la répartition des tâches se soit faite en fonction de la nature des travaux poursuivis dans les laboratoires allemands, les militaires se préoccupant 
surtout des questions scientifiques intéressant directement l'armée. Pourtant, en matière nucléaire, secteur d'intérêt capital pour les militaires, F. Joliot eut la haute main sur les contacts avec les laboratoires et les collègues allemands ${ }^{38}$.

Fonctionnement et évolution de la mission

Si la coopération entre militaires et membres de la mission du CNRS devait rester excellente $^{39}$, des difficultés surgirent avec l'administration civile de la zone. En effet, plusieurs directions du Gouvernement militaire mis en place à l'été 1945 disposaient d'un bureau responsable de la recherche dans leur secteur, ce qui généra parfois des conflits de compétence ${ }^{40}$. Ainsi la Production industrielle annula-t-elle d'emblée bon nombre d'autorisations d'enlèvement accordées au CNRS au profit d'industriels français. Il fallut des interventions réitérées de F. Joliot et L. Couffignal pour que la mission obtînt l'attribution de machines-outils pour l'enseignement technique français ${ }^{41}$. Parla suite, la mission du CNRS fut en contact étroit avec les deux services de la Production industrielle responsables des recherches techniques et brevets et de l'administration française de l'IG Farben. Ils lui signalaient des savants ou des procédés de fabrication intéressants et la consultaient sur des questions purement scientifiques. Avec la Santé publique (sections médecine et pharmacie), la mission échangeait des informations concernant les recherches, savants et produits pharmaceutiques allemands. Avec la direction de l'Agriculture, les relations furent toujours bonnes, mais de moins en moins fréquentes. Des contacts existaient aussi entre la mission du CNRS et les services spéciaux (services secrets) français. Ces services jouaient un rôle clé pour l'envoi de techniciens en France et pour l'octroi de passeports aux savants allemands voulant assister à des congrès en France. La mission entretint d'excellentes relations avec ces services, échangeant des renseignements sur la valeur scientifique des savants et techniciens allemands, la confiance qu'on pouvait leur accorder et l'intérêt de leurs découvertes scientifiques ${ }^{42}$.

18 En revanche, les relations furent plus tumultueuses avec la Direction de l'Éducation publique (DEP) dirigée par le germaniste Raymond Schmittlein. La DEP disposait d'un bureau chargé de contrôler la recherche universitaire, géré par R. Rouillier ${ }^{43}$. Responsable en zone française de tout ce qui relevait du ministère de l'Éducation nationale en France, R. Schmittlein entendait avoir la haute main sur la recherche fondamentale menée tant dans les universités que dans les instituts de l'ancienne Kaiser-Wilhelm-Gesellschaft. Aussi contestait-il l'intégration de la mission du CNRS aux services dépendant des autorités militaires ${ }^{44}$. D'incessants conflits devaient opposer la mission et la DEP au sujet de la nature des liens entre ex-KWI et universités, des nominations d'universitaires, du contrôle des laboratoires.

À partir de l'été 1945, F. Joliot assigna à la mission des tâches plus diversifiées. Outre la récupération de matériel, la mission devait acheter sur place et à très bon prix du matériel neuf souvent introuvable en France. Fin 1945, un fonds de 18 millions de francs, prélevé sur le budget du Centre en accord avec le ministère des Finances, avait été attribué au chef de la mission à cet effet ${ }^{45}$. Jusqu'au printemps 1946, le matériel récupéré n'avait coûté que le prix de son transport vers la France, puis les Douanes intervinrent dans le processus contraignant le CNRS à payer le matériel allemand qu'il importait. Il devint alors plus intéressant d'acquérir du matériel neuf, son coût restant bien inférieur à celui du marché français ${ }^{46}$. Les achats furent effectués, non seulement en zone française, mais aussi dans les zones alliées et en Autriche. 
Tout en poursuivant son travail d'information scientifique, la mission devait commencer à prendre en charge et à contrôler les laboratoires et savants allemands. Depuis l'été 1945, les occupants avaient jeté les bases d'une politique de contrôle de la recherche en Allemagne. Après avoir énoncé le principe du contrôle à la conférence de Potsdam, ils tentèrent de définir une politique commune en promulguant la loi $\mathrm{n}^{\circ} 25$ en avril $1946^{47}$. S'inscrivant dans le cadre du désarmement et de la démilitarisation, elle visait à interdire la recherche qui pouvait aboutir à des applications militaires et prévoyait un contrôle très strict de la recherche fondamentale ${ }^{48}$. Cette prise en charge de la recherche allemande recouvrit deux formes pour chacun des quatre occupants : 1 ) le contrôle de la recherche allemande autorisée (ce qui n'excluait pas d'en tirer profit). F. Joliot estimait qu'il ne fallait permettre les recherches allemandes "que dans les domaines où nous sommes assez forts et avons par conséquent un réel moyen de contrôle ${ }^{49} "$;2) l'exploitation directe du potentiel scientifique allemand. Se posait alors la question du transfert des chercheurs allemands vers l'étranger.

Dès le printemps 1945, les "grands Alliés" s'étaient lancés dans la "chasse aux cerveaux". Les Soviétiques recherchaient surtout des spécialistes de l'atome et les Américains des experts en balistique. À l'été 1945, ces derniers avaient emmené cent vingt-neuf spécialistes outre-Atlantique. En 1946, ils entreprirent l'opération Paperclip, projetant de faire venir 1000 spécialistes allemands aux États-Unis, d'une part pour les mettre hors de portée des Soviétiques, d'autre part pour profiter pleinement des transferts de technologie. Ils parvinrent à n'en faire venir que six cents environ ${ }^{50}$, parmi lesquels d'anciens nazis convaincus ${ }^{51}$. Les Britanniques avaient recruté quelque cinq cents experts allemands. Plusieurs milliers, voire dizaines de milliers d'Allemands, avaient été emmenés travailler en URSS jusqu'en 1949, la plupart contre leur gré. Les Occidentaux en revanche se refusèrent de faire venir chez eux des savants allemands par la contrainte ${ }^{52}$.

Pour la partie française, la volonté de profiter de la manne des savants, ingénieurs et techniciens allemands est attestée dès la mi-mai 1945 par un mémorandum de l'étatmajor de la Défense nationale au général de Gaulle, insistant sur le recrutement entrepris par les Alliés et conseillant "d'attirer les travailleurs bénévoles (sic; comprendre volontaires) dans les laboratoires français ". C'était la thèse des militaires, à laquelle de Gaulle adhéra, à condition que l'on ne fît venir en France que les experts de grande valeur ${ }^{53}$. Au début de l'Occupation, F. Joliot ne partageait pas cette thèse. Il se prononça au contraire pour l'exploitation in situ de la recherche allemande ${ }^{54}$. F. Joliot puis son successeur $G$. Tessier refusèrent de mettre les savants allemands en concurrence directe avec des chercheurs français ${ }^{55}$. Une note du 7 août 1946, rédigée par L. Cagniard, précisait en termes abrupts la position de la mission : "Il ne semble pas qu'un technicien ou savant, parce qu'il est allemand, soit obligatoirement un surhomme. Il ne faut pas se laisser gagner par un engouement dont nos alliés ont donné l'exemple, ni par le battage que les Allemands font autour de leurs personnes et de leurs travaux. Nous avons en France des quantités de jeunes chercheurs - sans compter les vétérans - qui pourraient certainement faire aussi bien que ces Allemands si l'on mettait à leur disposition les crédits et l'outillage qu'il faudrait bien donner à ces étrangers. Notre politique vis-à-vis des savants allemands doit consister plutôt à en extraire des renseignements aussi étendus que possible sur les idées nouvelles qu'ils ont eues, les résultats qu'ils ont obtenus et les méthodes qui leur ont permis d'atteindre ces résultats. Les considérer comme des vaches à lait qu'on abandonnera après les avoir 
traites ${ }^{56}$." Or, onze mois plus tard, prétextant vouloir éviter une grave crise de maind'œuvre, le CNRS modifia sa position et prit parti pour l'introduction de travailleurs allemands en France $^{57}$. Dans les faits, sous la pression de la concurrence interalliée, nombre de techniciens allemands avaient déjà été transférés en France par la direction de la Production industrielle ${ }^{58}$.

L'exploitation directe des chercheurs ne suffisait pas à assurer un transfert de technologie optimal. Aussi, dès 1945, les Alliés participèrent à la création ou à la remise en état, en Allemagne même, de laboratoires allemands dont les programmes de recherche les intéressaient tout spécialement. Soviétiques et Français y recoururent fréquemment. Ils obtenaient de meilleures informations des chercheurs allemands en les observant travailler sur du matériel performant (dont ils ne disposaient souvent pas eux-mêmes), entourés de leurs équipes ${ }^{59}$. En zone française, des commissions de spécialistes français furent mises en place. Elles devaient " associer scientifiquement le laboratoire allemand aux laboratoires similaires français, fixer aux Allemands un programme de recherches, approuver un budget de recherche [payés par les Allemands]... provoquer des visites de contrôle, trouver et prendre en charge des étudiants et de jeunes scientifiques français désir[ant] aller s'initier pendant un an ou deux aux méthodes allemandes ${ }^{60}$ " Ainsi, dès 1945, le laboratoire de recherche sur les métaux replié à Constance fut dirigé par une équipe française et les chercheurs allemands y furent encadrés par des Français “ avec lesquels ils n'entretenaient que des contacts de travail ${ }^{61} "$.

24 La présence de jeunes chercheurs français dans les laboratoires allemands fut une pratique très répandue (Christian Ythier au KWI de chimie, Georges Philbert au KWI de physique, Paul Fouché au KWI de biochimie). Outre la formation de cette nouvelle génération aux techniques récentes, ce système permettait d'assurer un certain contrôle des établissements allemands ${ }^{62}$. Les stagiaires semblent avoir été dirigés en priorité vers les instituts de l'ancienne KWG : sans aucun doute parce qu'ils étaient à la pointe de la recherche allemande, mais aussi parce que ces laboratoires constituaient des "atomes libres" depuis la dissolution de la KWG. La question du statut de ces instituts fut l'un des problèmes les plus difficiles à gérer pour la mission du CNRS. Alors que les Britanniques s'orientaient vers la reconstitution d'une société mère sous le nom de Max-Planck-Gesellschaft (MPG) ${ }^{63}$, le CNRS s'était prononcé en 1946 et 1947 contre la centralisation de la recherche allemande et la création de tout organisme de liaison entre les ex-KWI ${ }^{64}$. Ce refus de la centralisation correspondait parfaitement à la ligne directrice de la politique allemande de la France. Le Gouvernement militaire français et le CNRS cherchèrent une solution en rattachant administrativement les KWI aux universités. Ce rapprochement entre ex-KWI et universités devait se traduire d'une part par le transfert de ces laboratoires sur les sites universitaires (Tübingen et Mayence en particulier), d'autre part par la nomination des chercheurs les plus renommés à des postes de professeurs. Ainsi la mission du CNRS intervint-elle à de nombreuses reprises auprès des services de R. Schmittlein pour faire nommer tel ou tel savant à l'Université ${ }^{65}$. Elle semble n'avoir eu qu'une influence très modérée sur le recrutement universitaire. Certes, Fritz Strassmann, dont la candidature avait été personnellement soutenue par F. Joliot, fut nommé à l'université de Mayence, mais la mission ne réussit pas à faire nommer Wolfgang Gentner ${ }^{66}$, Siegfried Flügge, Gottfried von Droste et Wolfgang Riezler dans cette université ${ }^{67}$. Ces échecs s'expliquaient par le manque de coopération entre la mission et la DEP, par les intrigues entre savants 
allemands (rivalités entre universitaires et chercheurs de l'ex-KWG) ${ }^{6}$. À l'automne 1947, la situation des laboratoires de l'ancienne KWG restant très précaire, R. Schmittlein proposa la création dans chaque Land de la zone française d'organismes de recherche chargés de gérer budget et matériel. Mais les négociations n'aboutirent pas et le problème ne fut résolu qu'après la fondation de la République fédérale d'Allemagne. Les autorités françaises acceptèrent alors le regroupement des ex-KWI de zone française dans la nouvelle Max-Planck-Gesellschaft qu'elles avaient reconnues le 8 juillet $1949^{69}$. La guerre froide et le blocus de Berlin avaient beaucoup accéléré ce processus $^{70}$. Ainsi, les efforts du Gouvernement militaire pour empêcher toute centralisation de la recherche avaient échoué.

Pour mieux saisir le rôle de la mission du CNRS dans le contrôle de la recherche allemande, analysons la manière dont elle procéda dans le secteur qu'elle privilégia, celui de la recherche nucléaire.

Dès la première visite de F. Joliot, en juin 1945, le KWI de Tailfingen avait obtenu la protection de la mission du CNRS. Elle lui avait laissé son indépendance en lui évitant la tutelle directe d'un officier de contrôle. Cette décision résultait du prestige des savants de l'institut (O. Hahn, Lise Meitner et F. Strassmann y avaient découvert la fission de l'atome en 1938) et de leur non-compromission sous le III ${ }^{\mathrm{e}}$ Reich (L. Meitner avait été contrainte à l'exil et $\mathrm{F}$. Strassmann avait préféré renoncer à son habilitation que d'adhérer au parti national-socialiste) $)^{71}$.

La mission du CNRS fut bientôt chargée de nouer des liens entre les laboratoires allemands de physique nucléaire et le Commissariat à l'énergie atomique (CEA) pour lequel elle travaillait depuis sa fondation. En cela, elle ne changeait pas de patron : $F$ Joliot avait quitté la direction du CNRS pour celle du CEA en février 1946, et son adjoint F. Teissier l'avait remplacé à la tête du Centre ${ }^{72}$.

28 La première étape consistait à rassembler en un même lieu les instituts de l'ancienne Kaiser-Wilhelm-Gesellschaft travaillant sur l'atome (instituts de Tailfingen, Hechingen et Haigerloch $)^{73}$. Cette mesure visait à faciliter le contrôle par les autorités françaises et à éviter le départ des savants. Ce risque était grand étant donné les conditions de travail des laboratoires repliés en zone française ${ }^{74}$, et l'attrait que Göttingen, où la KWG renaissait sous le nom de MPG, exerçait sur les chercheurs allemands ${ }^{75}$. D'ailleurs, Josef Mattauch, directeur de l'institut depuis l'installation de O. Hahn à Göttingen, et F. Strassmann avaient déjà envisagé de l'y rejoindre. Français et Allemands étaient finalement parvenus à s'entendre sur un transfert vers Tübingen quand R. Schmittlein proposa de regrouper les principaux ex-KWI de zone française à Mayence. J. Mattauch et $\mathrm{F}$. Strassmann donnèrent leur accord ${ }^{76}$, mais les transferts du KWI de Haigerloch et de la section de physique nucléaire de l'Institut de Hechingen échouèrent ${ }^{77}$.

La deuxième étape visait à employer ces spécialistes allemands de l'atome "successivement ou en groupe dans les laboratoires du Commissariat ${ }^{78 "}$ ". En février 1946, F. Joliot avait même proposé à J. Mattauch, F. Strassmann et Arnold Flammersfeld de venir s'implanter en France avec leurs installations. Cette proposition, en contradiction avec les principes du physicien sur le maintien des savants allemands en Allemagne, témoigne de l'intérêt particulier de la France pour la recherche nucléaire, mais les trois chercheurs allemands refusèrent catégoriquement ${ }^{79}$.

La fin de la mission du CNRS en Allemagne et son bilan 

chercheurs en 1946, ils étaient tombés à dix en 1947 puis à deux depuis 1948. Ces deux derniers membres, J. Decombe et son adjoint R. Guillien, revinrent en France en octobre 1950. Au cours de leur dernière année en Allemagne, ils avaient dû gérer la liquidation de la mission. Dès 1949, les bureaux de Berlin, Innsbruck et Vienne avaient été fermés et la dissolution de la direction des missions techniques dont dépendait la mission était annoncée. Il fallut trouver un nouvel organisme auquel la rattacher. Après avoir été placée en février 1950 sous la coupe du groupe français de contrôle scientifique, lié à l'office militaire de sécurité, la mission fut dissoute à l'automne. Gaston Dupouy, physicien et directeur général du CNRS depuis le départ de F. Teissier en janvier 1950, souhaitait que le CNRS restât néanmoins informé de la recherche allemande. Aussi demanda-t-il au professeur Genevois, membre du groupe français de contrôle scientifique, de "mettre à profit [ses] occupations actuelles pour tenir le CNRS au courant des diverses activités scientifiques qu'[il] juge[rait] intéressant de [lui] signaler". G. Dupouy envisageait alors d'organiser des missions temporaires pour visiter des laboratoires ou assister à des manifestations scientifiques ${ }^{80}$.

31 Au moment du départ, J. Decombe dressa un bilan plutôt amer du travail effectué par la mission: "Le CNRS semble avoir déçu beaucoup de monde en se réduisant à une mission d'achat. On attendait de lui une collaboration technique et scientifique qui a été effective à un moment donné, mais qui, depuis plus d'un an, s'est réduite progressivement à zéro. ${ }^{81}$ " Pourtant, comme l'a montré Marie-France Ludmann-Obier, le bilan chiffré était globalement positif, les bénéfices sur les cessions de matériel et ventes de machines-outils étant très supérieurs aux frais généraux ${ }^{82}$. Fin 1946, plus de huit cents tonnes de matériel, machines-outils et appareils de laboratoires (surtout les microscopes électroniques) avaient été transférés en France, et quelque cinq cents machines-outils attribuées directement à l'enseignement technique. Les laboratoires de Bellevue, la station nationale de recherche Claude-Bonnier, les laboratoires de SaintCyr, l'Institut d'astrophysique et les laboratoires dépendant de l'université de Grenoble furent rééquipés en priorité avec le matériel récupéré en Allemagne. Plusieurs tonnes de matières premières presque introuvables à l'époque (laiton, aluminium, cuivre, acier inoxydable, nickel pur) furent attribuées au centre de Bellevue. La valeur du matériel récupéré, au $1^{\mathrm{er}}$ octobre 1946, était déjà évaluée à plus de 100000 francs $^{83}$. Le CNRS avait pu acheter au cours de la première année pour 8 millions de francs de matériel neuf dont la valeur réelle était quatre fois supérieure. Il s'agissait essentiellement d'équipement n'existant pas sur le marché français. Après la régularisation opérée à l'été 1946, le CNRS continua néanmoins à acquérir du matériel en Allemagne à des prix défiant toute concurrence. Fin 1949, la mission du CNRS, avec les FIAT anglaise et américaine, avait pratiquement bouclé l'édition complète des FIAT Reviews (études sur les travaux des scientifiques allemands menés entre 1939 et 1945). Plus de 85000 tomes avaient été publiés, tirés chacun à plus de 1000 exemplaires $^{84}$.

Cependant, au-delà des données chiffrées, le bilan de la mission à l'égard des instituts de l'ancienne KWG fut loin de correspondre aux objectifs qu'elle s'était initialement fixés: il n'y eut ni transfert massif des laboratoires vers la capitale rhénane, ni coopération particulière entre l'Institut de chimie de Mayence et les laboratoires de recherche en France. Le projet de démembrer la puissante KWG échoua en partie avec la réintégration de ses instituts dans la Max-Planch-Gesellschaft. Les objectifs français, en retard sur l'évolution politique, furent finalement modifiés et adaptés à la nouvelle 
situation allemande. Les premiers soubresauts de la guerre froide, puis plus encore la guerre de Corée, devaient conduire les Alliés occidentaux à passer du contrôle du désarmement allemand, qui comprenait une dimension scientifique, à celui du réarmement de l'Allemagne occidentale.

\section{NOTES}

1.Margaret Gowing, Dossier secret des relations atomiques entre Alliés, 1939-1945, Plon, Paris, 1965.

2.Nom de code de la mission.

3.Dirigée par le physicien Samuel Goudsmit, elle suivit la progression des troupes américaines depuis le débarquement en Normandie, fit une première moisson d'informations de premier ordre (plutôt rassurante pour les Alliés) à l'université de Strasbourg libérée, avant d'œuvrer en Allemagne. Voir Samuel Goudsmit, L'Allemagne et le secret atomique. La mission ALSOS, Fayard, Paris, 1948, p. 10.

4.Marie-France Ludmann-Obier, "La mission du CNRS en Allemagne (1945-1950) " in Cahiers pour l'Histoire du CNRS 1939-1989, 1989, n³ 3, p. 74.

5.Christoph Mick, “Forschen für die Siegermächte. Deutsche Naturwissenschaftler und Rüstungsingenieure nach dem Zweiten Weltkrieg ", in Dietrich Papenfuss \& Wolfgang Schieder (éd.) Deutsche Umbrüche im 20. Jahrhundert, Böhlau, Cologne/Weimar/Vienne, 2000, p. 432-433. Voir également le témoignage de Pjotr Nikitin, ancien officier d'université soviétique, Zwischen Dogma und gesundem Menschenverstand. Wie ich die Universitäten der deutschen Besatzungszone "sowjetisierte". Erinnerungen, Akademie Verlag, Berlin, 1997, p. 59-61. S'il rappelle comment les forces américaines s'emparèrent de nombreux chercheurs et techniciens allemands dans l'est de l'Allemagne, Nikitin passe complètement sous silence les démontages "scientifiques" et les transferts de "cerveaux" opérés par les Soviétiques dans leur zone d'occupation. 6.Werner Heisenberg et son équipe avaient orienté leurs recherches nucléaires sur ce qui devait être la voie de la pile atomique et non celle de la bombe. Cf. Bertrand Goldschmidt, Le Complexe atomique. Histoire politique de l'énergie nucléaire, Fayard, Paris, 1980, p. 22.

7.S. Goudsmit, op. cit., p. 109-131.

8.Ces savants, avec d'autres, furent ensuite remis aux autorités britanniques et internés près de Londres. C'est là qu'ils entendirent à la radio l'annonce d'un bombardement nucléaire sur Hiroshima. Leur incrédulité donna aux Britanniques une ultime confirmation du retard que les Allemands avaient accumulé. Voir D. Hoffmann, “ Operation Epsilon. Die Geheimdienstakten über die Internierung der deutschen Atomphysiker im englischen Farm Hall sind geöffnet ", Physikalische Blätter, 1992, n 12, p. 989-1001.

9.M. Bar-Zohar, La Chasse aux savants allemands, 1944-1960, Fayard, Paris, 1965, p. 72. 10.Monique Bordry \& Pierre Radvanyi, Histoires d'atomes, Belin, Paris, 1988, p. 181. 11.Marie-France Ludmann-Obier, Die Kontrolle der chemischen Industrie in der französischen Besatzungszone 1945-1949, Hase \& Koehler, Mayence, 1989, p. 136. 
12. “De la Kaiser Wilhelm à la Max Planck Gesellschaft ", texte adapté d'une conférence de l'ancien directeur de la MPG, H. A. Staab, prononcée le 13 juin 1986 à Aix-laChapelle, et d'un article de Robert Gerwin, “ 75 Jahre Max-Planck-Gesellschaft. Ein Kapitel deutscher Forschungsgeschichte " in Naturwissenschaftliche Rundschau, 1986, et in Revue d'Allemagne, 1988, t. 4, p. 378 et suiv.

13.Manfred Heinemann, “ Der Wiederaufbau der Kaiser-Wilhelm-Gesellschaft und die Neugründung der Max-Planck-Gesellschaft 1945-1949 " , in Rudolf Vierhaus \& Bernhard vom Brocke (éd.), Geschichte und Struktur der Kaiser-Wilhelm-/Max-Planck-Gesellschaft, DVA, Stuttgart, 1990, p. 408.

14.AN F 780283/39, document du 18 avril 1947, signé Koenig.

15.M. Heinemann, op. cit., p. 409.

16.M.-F. Ludmann-Obier. Die Kontrolle..., p. 136.

17.Jean-François Picard, La République des savants. La recherche française et le CNRS, Paris, Flammarion, 1990, p. 86-88. Diane Dosse, “Louis Rapkine (1904-1948) et la mobilisation scientifique de la France libre ”, thèse de doctorat, Paris VII-Denis-Diderot, décembre $1998,675 \mathrm{p}$.

18.Ibid., p. 96.

19.M.-F. Ludmann-Obier, “ La mission du CNRS... ”, p. 74. AN 780283/35, lettre de F. Joliot à Braillon, directeur de cabinet du ministre de l'Éducation nationale, 11 octobre 1945.

20.C. Mick, op. cit., p. 436-437. Voir aussi Alexandr Haritonow, Sowjetische Hochschulpolitik in Sachsen 1945-1949, Böhlau Verlag, Weimar/Cologne/Vienne, 1995, p. 100 et suiv.

21.J.-F. Picard, op. cit., p. 96.

22.M.-F. Ludmann-Obier, “ La mission du CNRS... ”, p. 74.

23. Corine Defrance, La Politique culturelle de la France sur la rive gauche du Rhin, Presses universitaires de Strasbourg, 1994, p. 87.

24.J.-F. Picard, op. cit., p. 96.

25.S. Goudsmit, op. cit., p. 105.

26.AN F 780283/44, note du 7 août 1946.

27.M.-F. Ludmann-Obier, “La mission du CNRS... ”, p. 74.

28. Cette antenne régionale était en fait chargée du Wurtemberg-Hohenzollern et du cercle de Lindau. La Bavière faisait partie de la zone d'occupation américaine, mais les Américains avaient accepté que le cercle bavarois de Lindau fût soustrait de leur zone au bénéfice des Français, de manière à créer un lien géographique entre les zones d'occupation françaises en Allemagne et en Autriche.

29.AN F 800284/55, “ Compte rendu de l'activité du CNRS de septembre 1944 à octobre $1945 "$.

30.AN F 800284/48, “ Rapport sur l'ensemble des missions en Allemagne effectuées par M. Vichnievsky", sans date.

31.AN F 800284/55, “Compte rendu de l'activité du CNRS de septembre 1944 à octobre $1945 "$.

32.AN F 800284/55, “Compte rendu de l'activité du CNRS de septembre 1944 à octobre $1945 "$.

33.AN F 800284/48, “ Rapport sur l'ensemble des missions en Allemagne effectuées par M. Vichnievsky", sans date.

34.AN F 800284/55, “ Compte rendu de l'activité du CNRS de septembre 1944 à octobre $1945 "$. 
35.AN F 800284/55, “ Compte rendu de l'activité du CNRS de septembre 1944 à octobre $1945 "$.

36.AN F 800284/115, “ Compte rendu... ”, 25 novembre 1946.

37.AN F 800284/55, “ Compte rendu de l'activité du CNRS de septembre 1944 à octobre $1945 "$.

38. Voir infra.

39.Marie-France Ludmann-Obier, “ Le contrôle de la recherche scientifique en zone française d'Occupation en Allemagne, 1945-1949 ", in Revue d'Allemagne, 1988, n 4, p. 404.

40.AN F 800284/115, “ Services français avec lesquels la mission CNRS en Allemagne est en rapport, situation en juillet 1949 ".

41.AN F800284, $\mathrm{n}^{\circ}$ 115, “ Compte rendu... ”, 25 novembre 1946.

42.AN F 800284/115, “ Services français avec lesquels la mission CNRS en Allemagne est en rapport, situation en juillet 1949 ".

43.AN F 800284/115, "Services français avec lesquels la mission CNRS en Allemagne est en rapport, situation en juillet 1949 ".

44.AN F 780283/35, “ Note pour Monsieur Joliot-Curie ”, 17 octobre 1945.

45.AN F 800284/55, “ Compte rendu de l'activité du CNRS de septembre 1944 à octobre $1945 "$

46.M.-F. Ludmann-Obier, “La mission du CNRS... ”, p. 80-81.

47.M.-F. Ludmann-Obier, "Le contrôle de la recherche scientifique... ”, p. 397. Si la loi $n^{\circ} 25$ avait été édictée par les quatre occupants, rappelons que le gouvernement français n'avait pas été invité à la conférence tripartite de Potsdam à l'été 1945. 48.Loi $n^{\circ} 25$, "Réglementation et contrôle de la recherche scientifique " du 29 avril 1946, publiée au Journal officiel du commandement en chef français en Allemagne, $\mathrm{n}^{\circ} 6,30$ avril 1946. Cette loi émana du Conseil de contrôle de Berlin, seule instance quadripartite en Allemagne.

49.AN F 780283/38, procès-verbal de la réunion tenue le 15 novembre 1947 au CNRS.

50.C. Mick, op. cit., p. 442-443.

51.Linda Hunt, L'Affaire Paperclip. La récupération des scientifiques nazis par les Américains 1945-1990, Stock, Paris, 1995. L. Hunt a mis en lumière le fait que l'opération Paperclip ne s'est pas terminée à la fin des années 1940, comme l'opinion publique américaine l'a trop longtemps cru, mais qu'elle s'est en fait poursuivie pendant toute la période de la guerre froide (au sens large) jusqu'en 1990. Les Américains auraient réussi à faire venir 1600 chercheurs et techniciens allemands aux États-Unis. Voir aussi Tom Bower, The Paperclip conspiracy. The Hunt for Nazi Scientists, Little, Brown \& Company, Boston/ Toronto, 1987.

52.C. Mick, op. cit., p. 442-443.

53.M.-F. Ludmann-Obier, “ Un aspect de la chasse aux cerveaux : les transferts de techniciens allemands en France, 1945-1949 ", in Relations internationales, n 46, 1986, p. 196-197.

54.AN F 780283/44, fiche du 9 juillet 1947, “Les techniciens allemands en France ”. 55.En 1948 encore, la mission du CNRS devait faire obstacle au transfert en France d'un chercheur allemand spécialiste du froid alors que ses collègues français dans la discipline manquaient de moyens : “Ils sont très mal payés. Ils sont par principe hostiles à la présence d'un chercheur allemand qui serait beaucoup mieux payé qu'eux ”, voir M.-E. Ludmann-Obier, “ La mission du CNRS... ”, p. 82.

56.AN F 780283/44, note du 7 août 1946. 
57.AN F 780283/44, fiche du 9 juillet 1947.

58.M.-F. Ludmann-Obier, “ Un aspect de la chasse aux cerveaux... ”, p. 195-208.

59.C. Mick, op. cit., p. 438-439.

60.AN F 800284/115, “ Compte rendu sur la mission du CNRS en Allemagne ”, 25 novembre 1946.

61.AN F 800284/55, “ Compte rendu de l'activité du CNRS de septembre 1944 à octobre $1945 "$.

62.AN F 800284/115, rapport sur les “ services français avec lesquels la mission CNRS en Allemagne est en rapport ", juillet 1949.

63.M. Heinemann, op. cit., p. 430 et suiv.

64.AN F 780283/38, procès-verbal de la réunion du 15 novembre 1947. Marie-France

Ludmann-Obier, “ Le contrôle de la recherche scientifique... ”, p. 413.

65.En principe, les universités, en vertu de l'autonomie universitaire, et les ministères de l'Éducation des Länder étaient responsables des nominations, mais l'occupant français se réserva la possibilité, dans des cas exceptionnels, de nommer lui-même des enseignants. Cf. Corine Defrance, Les Alliés occidentaux et les universités allemandes, 1945-1949, CNRS Éditions, Paris, 2000, p. 116-117.

66.Physicien, professeur à l'université de Fribourg. La mission du CNRS avait souhaité le nommer directeur de l'Institut de physique de Hechingen, dirigé par Schüller depuis le départ de W. Heisenberg (AN F 780283/39, lettre de Guillien à F Joliot, 8 février 1947). 67.L'université de Mayence ayant été fondée par l'occupant français en mai 1946, les autorités du gouvernement militaire disposaient d'une plus grande marge de manœuvre dans cet établissement, d'autant plus qu'au début de 1946, il fallut recruter près de cent enseignants. Cf. C. Defrance, La Politique culturelle..., p. 71 et suiv.

68.AN F 780283/38, lettre de L. Cagniard à R. Schmittlein du 15 juin 1946, lettres de Guillien à G. Teissier des 12 novembre 1947 et 3 mars 1948, et rapport de Guillien concernant le transfert des KWI à Mayence du 17 mars 1949.

69.C. Defrance, La Politique culturelle..., p. 275-276.

70.M. Heinemann, op. cit., p. 448.

71.Fritz Krafft, Im Schatten der Sensation, Leben und Wirken von Fritz Straßmann, Verlag Chemie, Weinheim, 1981, p. 341 et 408-409.

72.AN F 800284/115, “ Compte rendu sur la mission du CNRS en Allemagne ”, 25 novembre 1946.

73.AN F 780283/38, procès-verbal de la réunion tenue le 18 novembre 1947 au CNRS.

74.AN F 780283/38, compte rendu de la réunion du 15 novembre 1947.

75.M. Heinemann, op. cit., p. 430 et suiv., et CNRS, 780283/39, lettre de Guillien au CNRS, 18 octobre 1947.

76.C. Defrance, La Politique culturelle..., p. 91-93.

77.AN F 780283/38, lettre de Joliot au CNRS, 17 juillet 1946, rapports de Guillien des 3 mars 1948 et 30 janvier 1950 .

78.AN F 780283/38, lettre de F. Joliot à Pérès, 17 juillet 1946.

79.F. Krafft, op. cit., p. 136.

80.AN F 800284/115, lettre de G. Dupouy à Genevois, 6 juin 1950.

81.M.-F. Ludmann-Obier, “ La mission du CNRS... ”, p. 83.

82.Ibid.

83.AN F 800284/115, “Compte rendu...”, 25 novembre 1946.

84.AN F 800284/115, “ Rattachement de la mission CNRS en Allemagne ”, J. Decombe à André François-Poncet, haut-commissaire de France en Allemagne, 7 octobre 1949. 
INDEX

Mots-clés : seconde guerre mondiale, Allemagne

\section{AUTEUR}

CORINE DEFRANCE

Corine Defrance est chercheur au Centre d'études germaniques de Strasbourg, unité associée université de Strasbourg III / CNRS. Elle a publié Les alliés occidentaux et les universités allemandes, 1945-1949, CNRS Éditions, Paris, 2000. 